\title{
BALANCED CENTRAL SCHEMES FOR THE SHALLOW WATER EQUATIONS ON UNSTRUCTURED GRIDS*
}

\author{
STEVE BRYSON ${ }^{\dagger}$ AND DORON LEVY $\ddagger$
}

\begin{abstract}
We present a two-dimensional, well-balanced, central-upwind scheme for approximating solutions of the shallow water equations in the presence of a stationary bottom topography on triangular meshes.

Our starting point is the recent central scheme of Kurganov and Petrova (KP) for approximating solutions of conservation laws on triangular meshes. In order to extend this scheme from systems of conservation laws to systems of balance laws one has to find an appropriate discretization of the source terms. We first show that for general triangulations there is no discretization of the source terms that corresponds to a well-balanced form of the KP scheme. We then derive a new variant of a central scheme that can be balanced on triangular meshes.

We note in passing that it is straightforward to extend the KP scheme to general unstructured conformal meshes. This extension allows us to recover our previous well-balanced scheme on Cartesian grids. We conclude with several simulations, verifying the second-order accuracy of our scheme as well as its well-balanced properties.
\end{abstract}

Key words. shallow water equations, central schemes, balance laws, unstructured grids

AMS subject classifications. Primary, 65M06; Secondary, 76B15

DOI. $10.1137 / 040605539$

1. Introduction. We consider a flow in a two-dimensional channel with a bottom elevation given by $B(\vec{x})$, where $\vec{x}=(x, y)$. Let $H(\vec{x}, t)$ represent the fluid depth above the bottom, and let $\vec{u}(\vec{x}, t)=(u(\vec{x}, t), v(\vec{x}, t))$ be the fluid velocity. The top surface at any time $t$ is denoted by $w(\vec{x}, t)=B(\vec{x})+H(\vec{x}, t)$.

The shallow water equations, introduced by Saint-Venant in [24] (see also [10]), are commonly used to model flows in rivers or coastal areas. When written in terms of the top surface $w$ and the momentum $(H u, H v)$, these equations are of the form

$$
\left\{\begin{array}{l}
w_{t}+(H u)_{x}+(H v)_{y}=0 \\
(H u)_{t}+\left[\frac{(H u)^{2}}{w-B}+\frac{1}{2}(w-B)^{2}\right]_{x}+\left[\frac{(H u)(H v)}{w-B}\right]_{y}=-g(w-B) B_{x} \\
(H v)_{t}+\left[\frac{(H u)(H v)}{w-B}\right]_{x}+\left[\frac{(H v)^{2}}{w-B}+\frac{1}{2}(w-B)^{2}\right]_{y}=-g(w-B) B_{y}
\end{array}\right.
$$

This choice of variables is particularly suitable for dealing with stationary steady-state solutions (see $[14,23]$ for details). For simplicity we fix the gravitational constant, $g$, from now on to be $g=1$.

* Received by the editors March 23, 2004; accepted for publication (in revised form) November 18, 2004; published electronically October 7, 2005.

http://www.siam.org/journals/sisc/27-2/60553.html

${ }^{\dagger}$ Program in Scientific Computing/Computational Mathematics, Stanford University, Stanford, CA 94305 and the NASA Advanced Supercomputing Division, NASA Ames Research Center, Moffett Field, CA 94035-1000 (bryson@nas.nasa.gov).

${ }^{\ddagger}$ Department of Mathematics, Stanford University, Stanford, CA 94305-2125 (dlevy@math. stanford.edu). The work of this author was supported in part by the National Science Foundation Career Grant DMS-0133511. 
In this work we are interested in approximating solutions of (1.1) on triangular meshes. Our goal is to investigate how to adapt the semidiscrete central schemes on triangular meshes that were recently introduced by Kurganov and Petrova in [16] to this problem. We are interested in deriving a discretization of the source terms in (1.1) that preserves stationary steady-state solutions, as such solutions play an important role in the dynamics of (1.1). While such a balance is not pursued for nonstationary shallow water problems, our simulations indicate that our method works well for such problems.

Central schemes for conservation laws have become popular in recent years as a tool for approximating solutions for multidimensional systems of hyperbolic conservation laws. Like other Godunov-type schemes, central schemes are based on a three-step procedure: a reconstruction step in which an interpolant is reconstructed from previously computed cell averages; an evolution step in which this interpolant is evolved exactly in time according to the equations; and a projection step in which the solution is projected back to cell averages. When compared with other methods, central schemes are particularly appealing since they do not require any Riemann solvers and systems can be solved componentwise.

A first-order prototype of central schemes is the Lax-Friedrichs scheme [8]. A second-order extension is due to Nessyahu and Tadmor [21]. Extensions to two dimensions are due to Arminjon and Viallon [1] and Jiang and Tadmor [13]. By estimating bounds on the local speeds of propagation of information from discontinuities, it is possible to pass to the semidiscrete limit (see $[15,17]$ and the references therein). There are several extensions of central schemes to unstructured grids. A fully discrete method is due to Arminjon, Viallon, and Madrane [2]; a recent semidiscrete scheme was proposed by Kurganov and Petrova in [16]. Balanced central schemes for shallow water equations on Cartesian grids are due to Russo in the fully discrete framework [23] (see also [20]) and to Kurganov and Levy in the semidiscrete framework [14].

There are many approaches to approximating solutions of (1.1). We refer the reader to, e.g., [3, 4, 7, 9, 11, 18, 19, 22] and the references therein. An equilibrium technique for balancing scalar conservation laws is presented in $[5,6]$. Our goal in this paper is to show that balancing is also possible with central schemes. We would like to emphasize that this is the first time in which the balancing issues are treated for central schemes on unstructured grids.

The paper is organized as follows. We start in section 2 with a brief overview of the Kurganov-Petrova (KP) central scheme on triangular meshes. We note that this scheme is not limited to triangular meshes and it can be equally well applied to general unstructured grids. We also make the necessary adjustments to incorporate source terms into the scheme. We proceed in section 3 with the discretization of the cell averages of the source terms for the shallow water equations. The goal is to find a discretization such that the scheme will preserve stationary steady states, i.e., zero velocities and a flat surface. We show that on general unstructured meshes, there is no discretization of the source terms in the shallow water equations that provide a well-balanced form of the KP scheme. We then proceed by showing how to modify the original scheme in such a way that it is possible to obtain a well-balanced discretization of the source terms. We conclude in section 4 with numerical examples that demonstrate the accuracy of our scheme as well as its well-balanced property.

2. Central schemes for balance laws on unstructured grids. We consider the two-dimensional balance law,

$$
u_{t}+f(u)_{x}+g(u)_{y}=S(u, x, y, t),
$$




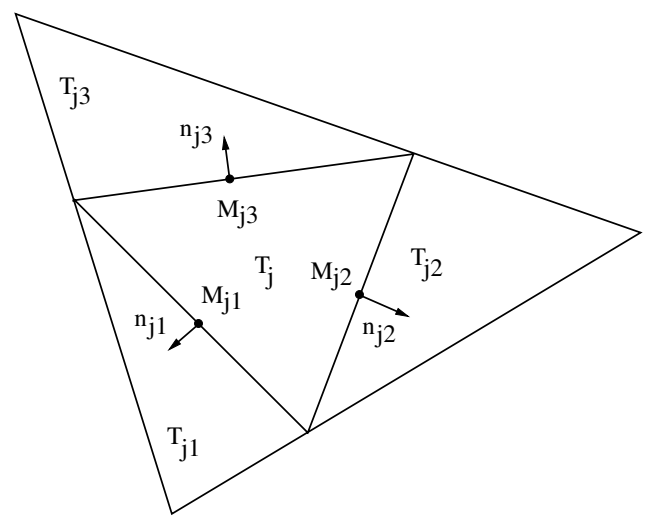

FIG. 2.1. The triangular grid.

subject to the initial data $u(x, y, 0)=u_{0}(x, y)$. We are interested in approximating solutions of (2.1) that are computed in terms of cell averages on a fixed unstructured conformal grid. To simplify this exposition we first consider the scalar case. The scheme that is described below can be easily extended componentwise to systems of balance laws. We will make this obvious extension later on when dealing with the specific problem of the system of shallow water equations (1.1).

We focus our attention on the central scheme on triangular meshes that was recently derived in [16]. We briefly overview the derivation of this scheme in the setup of conservation laws of the form

$$
u_{t}+f(u)_{x}+g(u)_{y}=0 .
$$

We assume a conformal triangulation of the domain consisting of cells $T_{j}$ of area $\left|T_{j}\right|$. The neighboring cells to $T_{j}$ are denoted by $T_{j k}, k=1,2,3$, while the edge that is joint between $T_{j}$ and $T_{j k}$ is denoted by $E_{j k}$ and is assumed to be of length $h_{j k}$. We also denote the outward unit normal to $T_{j}$ on the $k$ th edge as $n_{j k}$, and denote the midpoint of $E_{j k}$ as $M_{j k}$ (see Figure 2.1).

We assume that the cell averages on all the cells $\left\{T_{j}\right\}$ are known at time $t^{n}$,

$$
\bar{u}_{j}^{n} \approx \frac{1}{\left|T_{j}\right|} \int_{T_{j}} u\left(\vec{x}, t^{n}\right) d \vec{x}
$$

and reconstruct a piecewise polynomial

$$
\tilde{u}^{n}(x, y)=\sum_{j} u_{j}^{n}(x, y) \chi_{j}(x, y) .
$$

Here $u_{j}^{n}(x, y)$ is a two-dimensional polynomial that is yet to be determined and $\chi_{j}(x, y)$ is the characteristic function of the cell $T_{j}$. To simplify the notations we omit the time-dependence in $u_{j}(x, y)$. We also denote by $u_{j k}(x, y)$ the polynomial that is reconstructed in the cell $T_{j k}$.

Discontinuities in the interpolant $u_{j}$ along the edges of $T_{j}$ propagate with a maximal inward velocity $a_{j k}^{\text {in }}$ and a maximal outward velocity $a_{j k}^{\text {out }}$. These velocities can be estimated (for convex fluxes in the scalar case) as

$$
\begin{aligned}
a_{j k}^{\text {in }}\left(M_{j k}\right) & =-\min \left\{\nabla F\left(u_{j}\left(M_{j k}\right)\right) \cdot n_{j k}, \nabla F\left(u_{j k}\left(M_{j k}\right)\right) \cdot n_{j k}, 0\right\}, \\
a_{j k}^{\text {out }}\left(M_{j k}\right) & =\max \left\{\nabla F\left(u_{j}\left(M_{j k}\right)\right) \cdot n_{j k}, \nabla F\left(u_{j k}\left(M_{j k}\right)\right) \cdot n_{j k}, 0\right\},
\end{aligned}
$$


where $F=(f, g)$. These local speeds of propagation are then used to determine evolution points that are away from the propagating discontinuities. An exact evolution of the reconstruction at these evolution points is followed by an intermediate piecewise polynomial reconstruction and finally projected back onto the original cells, providing the cell averages at the next time step $\bar{u}_{j}^{n+1}$. Further details can be found in [16].

A semidiscrete scheme is then obtained at the limit

$$
\frac{d}{d t} \bar{u}_{j}^{n}=\lim _{\Delta t \rightarrow 0} \frac{\bar{u}_{j}^{n+1}-\bar{u}_{j}^{n}}{\Delta t} .
$$

Most of the terms on the right-hand side (RHS) of (2.5) vanish in the limit as $\Delta t \rightarrow 0$. The only quantity that has to be determined is a quadrature rule for the integrals of the flux functions over the edges of the cells. If we assume a Gaussian quadrature with $m$ nodes

$$
\int_{0}^{1} \varphi(x) d x \approx \sum_{s=1}^{m} c_{s} \varphi\left(x_{s}\right)
$$

which is scaled to $h_{j k}$, and denote the quadrature points on $E_{j k}$ as $G_{j k}^{s}$, the KP scheme for triangular meshes is

$$
\begin{gathered}
\frac{d \bar{u}_{j}}{d t}=-\frac{1}{\left|T_{j}\right|} \sum_{k=1}^{3} \frac{h_{j k}}{a_{j k}^{\text {in }}+a_{j k}^{\text {out }}} \sum_{s=1}^{m} c_{s}\left[\left(a_{j k}^{\text {in }} F\left(u_{j k}\left(G_{j k}^{s}\right)\right)+a_{j k}^{\text {out }} F\left(u_{j}\left(G_{j k}^{s}\right)\right)\right) \cdot n_{j k}\right. \\
\left.-a_{j k}^{\text {in }} a_{j k}^{\text {out }}\left(u_{j k}\left(G_{j k}^{s}\right)-u_{j}\left(G_{j k}^{s}\right)\right)\right]
\end{gathered}
$$

where $F=(f, g)$. If the fluxes are integrated with a midpoint quadrature (as suggested in [16]) and we use the notation $u_{j k}^{\text {out }}:=u_{j k}\left(M_{j k}\right), u_{j k}^{\text {in }}:=u_{j}\left(M_{j k}\right), F_{j k}^{\text {in }}:=F\left(u_{j k}^{\text {in }}\right)$, and $F_{j k}^{\text {out }}:=F\left(u_{j k}^{\text {out }}\right)$, the semidiscrete scheme (2.6) becomes

$$
\frac{d \bar{u}_{j}}{d t}=-\frac{1}{\left|T_{j}\right|} \sum_{k=1}^{3} \frac{h_{j k}}{a_{j k}^{\text {in }}+a_{j k}^{\text {out }}}\left[\left(a_{j k}^{\text {in }} F_{j k}^{\text {out }}+a_{j k}^{\text {out }} F_{j k}^{\text {in }}\right) \cdot n_{j k}-a_{j k}^{\text {in }} a_{j k}^{\text {out }}\left(u_{j k}^{\text {out }}-u_{j k}^{\text {in }}\right)\right]
$$

A basic observation that will be used below is that the semidiscrete scheme (2.7) is valid for any conformal grid, not necessarily triangular. All that one has to do is to make the suitable adjustments in the notations (e.g., $\left|T_{j}\right|$ being the area of the cell $T_{j}$ regardless of the shape of that cell) and replace the sum over the three edges of the triangle by a sum over the $N_{j}$ edges of each cell $T_{j}$. When approximating solutions to balance laws of the form (2.1) the scheme has one additional term due to the source term $S(u, x, y, t)$, i.e.,

$$
\begin{aligned}
\frac{d \bar{u}_{j}}{d t}(t)= & -\frac{1}{\left|T_{j}\right|} \sum_{k=1}^{N_{j}} \frac{h_{j k}}{a_{j k}^{\text {in }}+a_{j k}^{\text {out }}}\left[\left(a_{j k}^{\text {in }} F_{j k}^{\text {out }}+a_{j k}^{\text {out }} F_{j k}^{\text {in }}\right) \cdot n_{j k}-a_{j k}^{\text {in }} a_{j k}^{\text {out }}\left(u_{j k}^{\text {out }}-u_{j k}^{\text {in }}\right)\right] \\
& +\bar{S}_{j}(t) .
\end{aligned}
$$

Here

$$
\bar{S}_{j} \approx \frac{1}{\left|T_{j}\right|} \int_{T_{j}} S(u, \vec{x}, t) d \vec{x}
$$


is a discretization of the cell average of the source term that should be obtained with an appropriate quadrature. It is the discretization of (2.9) that serves as the topic for the next section.

Remark. The above derivation carries on to systems of balance laws componentwise. In this case the maximal speeds of propagation can be estimated (for convex fluxes) as

$$
\begin{aligned}
a_{j k}^{\text {in }}\left(M_{j k}\right) & =-\min \left\{\lambda_{1}\left[J\left(u_{j}\left(M_{j k}\right)\right) \cdot n_{j k}\right], \lambda_{1}\left[J\left(u_{j k}\left(M_{j k}\right)\right) \cdot n_{j k}\right], 0\right\}, \\
a_{j k}^{\text {out }}\left(M_{j k}\right) & =\max \left\{\lambda_{N}\left[J\left(u_{j}\left(M_{j k}\right)\right) \cdot n_{j k}\right], \lambda_{N}\left[J\left(u_{j k}\left(M_{j k}\right)\right) \cdot n_{j k}\right], 0\right\},
\end{aligned}
$$

where $J\left(u_{j}\left(M_{j k}\right)\right)$ is the Jacobian of the flux $F=(f, g)$ evaluated at $M_{j k}$, and $\lambda_{1}<\cdots<\lambda_{N}$ are its $N$ eigenvalues.

3. A well-balanced scheme. In this section we present a scheme for approximating the solution of (1.1) which is balanced via a discretized average of the source terms. In section 3.1 we show that the KP scheme (2.8) cannot be balanced using a straightforward discretization of the source terms on general conformal unstructured meshes. Using the insights gained from this analysis, in section 3.2 we present a new scheme based on (2.6), which does allow such balance on general conformal triangular meshes.

3.1. Balancing the KP scheme. Our goal now is to look for a discretization of the source term (2.9) such that the scheme (2.8) will preserve stationary steady-state solutions. Hence, we assume zero velocities, $u=v=0$, and a constant surface, i.e., $w=$ Const.

Since the first equation in (1.1) is homogeneous, we start by considering the second equation. Similar analysis applies to the third equation. We are therefore looking for a discretization of the average of the source term

$$
-(w-B) B_{x}
$$

over the cell $T_{j}$. The velocities are zero and therefore the only nonzero component of the flux in (1.1) is

$$
f=\frac{1}{2}(w-B)^{2} .
$$

This means that in order for the scheme (2.8) to preserve stationary steady-state solutions, the average of the source term (3.1) over the cell $T_{j}$ has to be discretized such that, for $f$ given in (3.2),

$$
0=-\frac{1}{\left|T_{j}\right|} \sum_{k=1}^{N_{j}} \frac{h_{j k}}{a_{j k}^{\text {in }}+a_{j k}^{\text {out }}}\left[\left(a_{j k}^{\text {in }} f_{j k}^{\text {out }}+a_{j k}^{\text {out }} f_{j k}^{\text {in }}\right) n_{j k, x}\right]+\bar{S}_{j} .
$$

Here $n_{j k, x}$ is the component of the normal $n_{j k}$ in the $x$-direction.

The eigenvalues of the Jacobian of the system (1.1) are $u \pm \sqrt{w-B}$ and $u$, which are $\pm \sqrt{w-B}$ and 0 in the case of zero velocities. If we assume that $w$ is constant and that the point values of $B$ are known, the one-sided velocities satisfy $a_{j k}^{\text {in }}=a_{j k}^{\text {out }}$. Under these assumptions the condition (3.3) can be rewritten as

$$
\bar{S}_{j}=\frac{1}{\left|T_{j}\right|} \sum_{k=1}^{N_{j}} h_{j k}\left[\left(\frac{f_{j k}^{\text {out }}+f_{j k}^{\text {in }}}{2}\right) n_{j k, x}\right]=\frac{1}{\left|T_{j}\right|} \sum_{k=1}^{N_{j}} \frac{h_{j k}}{2}\left[\left(w-B_{j k}\right)^{2} n_{j k, x}\right],
$$


where $B_{j k}=B\left(M_{j k}\right)$.

We now assume a discretization of the cell average of the source (3.1) of the form

$$
\bar{S}_{j}=-\sum_{k=1}^{N_{j}} g_{j k}\left(w-B_{j k}\right) \mathcal{D},
$$

where $\mathcal{D} \approx B_{x}$, and $g_{j k}$ are yet to be determined. To simplify the notations we denote $m_{k}=h_{j k} n_{j k, x}$. It is easy to check that $\sum_{k=1}^{N_{j}} m_{k}=0$ and hence in order for the representation (3.5) to be consistent with (3.4) we must have

$$
\mathcal{D}=-\frac{1}{2\left|T_{j}\right|} \frac{\sum_{k=1}^{N_{j}} m_{k} B_{j k}\left(B_{j k}-2 w\right)}{\sum_{k=1}^{N_{j}} g_{j k}\left(w-B_{j k}\right)} .
$$

Since $\mathcal{D}$ in (3.6) should not be a function of $w$ we are seeking constants $a_{k}$ such that

$$
\sum_{k=1}^{N} m_{k} B_{k}\left(B_{k}-2 w\right)=\left[\sum_{k=1}^{N} g_{k}\left(w-B_{k}\right)\right]\left[\sum_{k=1}^{N} a_{k} B_{k}\right],
$$

where for simplicity we omit the obvious $j$-dependence from all the notations. Equation (3.7) can be rewritten as

$$
\left\{\begin{array}{l}
-2 \sum_{k=1}^{N} m_{k} B_{k}=\sum_{k=1}^{N} g_{k} \sum_{k=1}^{N} a_{k} B_{k}, \\
\sum_{k=1}^{N} m_{k} B_{k}^{2}=-\sum_{k=1}^{N} g_{k} B_{k} \sum_{k=1}^{N} a_{k} B_{k} .
\end{array}\right.
$$

The coefficients of the powers of $B_{k}$ in (3.8) produce the following system of equations:

$$
\begin{cases}-2 m_{i}=a_{i} \sum_{k=1}^{N} g_{k}, & i=1, \ldots, N, \\ m_{i}=-a_{i} g_{i}, & i=1, \ldots, N, \\ a_{i} g_{j}=-a_{j} g_{i}, & i \neq j, i, j=1, \ldots, N .\end{cases}
$$

Finally, from (3.9) we have

$$
g_{i}=\frac{1}{2} \sum_{k=1}^{N} g_{k}, \quad i=1, \ldots, N .
$$

Equation (3.10) can generally hold only for $N=2$, which means that one can expect to be able to balance the scheme (2.8) for stationary steady-state solutions only in cells that have two edges that contribute to the flux in the $x$-direction. This obviously excludes most meshes. There are two cases of special interest.

1. When the mesh is composed of triangles with one side that is parallel to the $x$-axis (see Figure 3.1), each cell has only two edges that contribute to the flux in the $x$-direction. In this case the system (3.9) can be solved. This result will enable us to introduce in section 3.2 a modification of the scheme (2.8) that can be balanced on general meshes. Due to symmetry considerations, such a mesh will not satisfy the balance conditions in the $y$-direction (that come from the third equation in (1.1)) unless all triangles are right triangles that are aligned with both coordinate axes. 


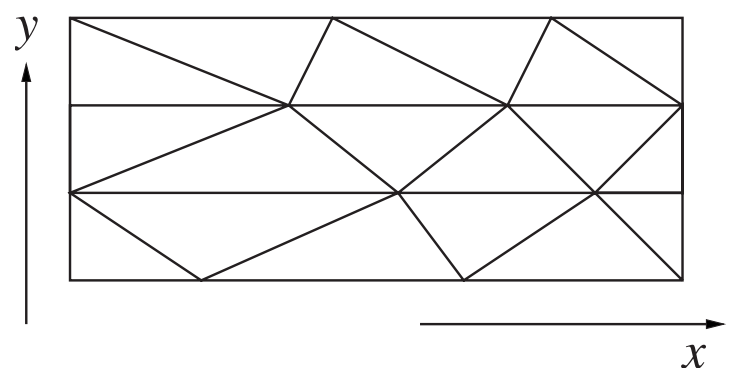

FIG. 3.1. An admissible triangular mesh.

2. The scheme can be balanced in both directions in the very special case of Cartesian grids. This corresponds to the case previously solved in [14]. The results of [14] when viewed from the point of view of the system (3.9) amount to the equality

$$
B_{1}\left(B_{1}-2 w\right)-B_{2}\left(B_{2}-2 w\right)=\left(2 w-B_{1}-B_{2}\right)\left(B_{2}-B_{1}\right) .
$$

Remark. If we assume a more general discretization of the cell average of (3.1) of the form

$$
\bar{S}_{j}=-\sum_{k=1}^{N} g_{k}\left(w-B_{k}\right) \sum_{k^{\prime}=1}^{N} a_{k k^{\prime}} B_{k^{\prime}},
$$

then the system (3.9) is replaced by (denoting $\tilde{m}_{k}=m_{k} /\left(2\left|T_{j}\right|\right)$ )

$$
\begin{cases}-2 \tilde{m}_{k}=\sum_{k^{\prime}=1}^{N} g_{k^{\prime}} a_{k^{\prime} k}, & k=1, \ldots, N, \\ \tilde{m}_{k}=-g_{k} a_{k k}, & k=1, \ldots, N, \\ g_{k} a_{k k^{\prime}}=-g_{k^{\prime}} a_{k^{\prime} k}, & k \neq k^{\prime}, k, k^{\prime}=1, \ldots, N .\end{cases}
$$

The system (3.12) can be solved in the general case (unlike the system (3.9)). For example, in the case of a triangular grid $(N=3)$ one possible solution (assuming $\tilde{m}_{1} \neq 0$ and $\left.\tilde{m}_{3}+2 \tilde{m}_{2} \neq 0\right)$ is

$$
g=\left(\begin{array}{c}
-\tilde{m}_{1} \\
\tilde{m}_{2}+\frac{\tilde{m}_{3}}{2} \\
\frac{\tilde{m}_{3}}{2}
\end{array}\right), \quad a=\left(\begin{array}{ccc}
1 & \frac{\tilde{m}_{3}+2 \tilde{m}_{2}}{2 \tilde{m}_{1}} & \frac{\tilde{m}_{3}}{2 \tilde{m}_{1}} \\
1 & -\frac{2 \tilde{m}_{2}}{2 \tilde{m}_{2}+\tilde{m}_{3}} & -\frac{\tilde{m}_{3}}{2 \tilde{m}_{2}+\tilde{m}_{3}} \\
1 & 1 & -2
\end{array}\right) .
$$

If $\tilde{m}_{1}=0$, the expression (3.13) can be replaced, e.g., by

$$
g=\left(\begin{array}{c}
0 \\
\tilde{m}_{2}+\frac{\tilde{m}_{3}}{2} \\
\frac{\tilde{m}_{3}}{2}
\end{array}\right), \quad a=\left(\begin{array}{ccc}
0 & \frac{\tilde{m}_{3}+2 \tilde{m}_{2}}{\tilde{m}_{3}} & 1 \\
1 & -\frac{2 \tilde{m}_{2}}{2 \tilde{m}_{2}+\tilde{m}_{3}} & -\frac{\tilde{m}_{3}}{2 \tilde{m}_{2}+\tilde{m}_{3}} \\
1 & 1 & -2
\end{array}\right) .
$$

If $\tilde{m}_{3}+2 \tilde{m}_{2}=0$, a permutation of the numbering of the sides of the triangle will provide a solution. Other solutions of (3.12) exist.

While formally being able to balance the scheme with the expressions (3.13) and (3.14), it remains unclear in what sense $\sum_{k^{\prime}} a_{k k^{\prime}} B_{k^{\prime}}$ approximates the derivative $B_{x}$. In other words, it is not obvious that the consistency of this discretization can be established. We therefore consider this approach unsatisfactory. 

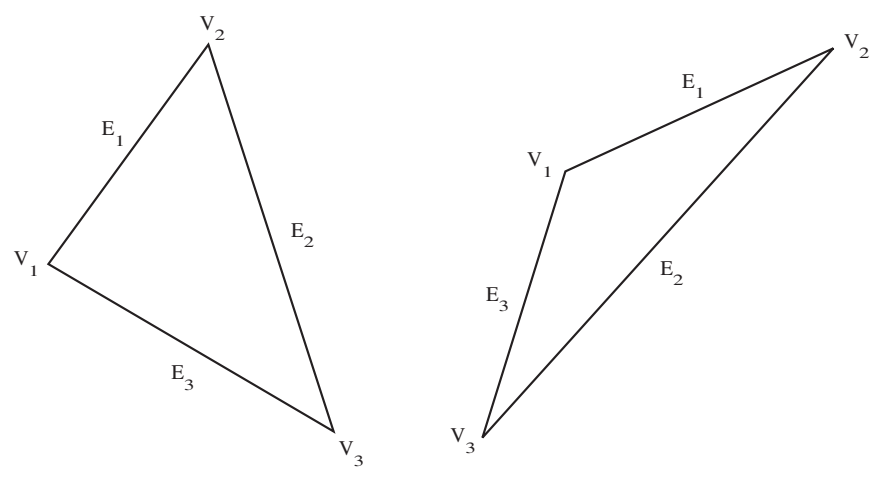

FIG. 3.2. The two types of triangles. Left: type 1. Right: type 2.

3.2. A new well-balanced scheme. The analysis in section 3.1 tells us that balancing the $x$-derivative flux term requires cells that have only 2 edges which have normals with nonzero $x$-components (see Figure 3.1 ). This can be accomplished by splitting our cells into subcells and solving the equation on these subcells. Similarly, the $y$-derivative flux term can be balanced by a different splitting of the cells. We now derive a new method based on this observation.

We focus on conformal triangular grids. Our ideas can be easily extended to other conformal unstructured meshes. In order to create a balanced scheme for (1.1), we propose to decompose every triangle $T_{j}$ into several triangles as explained below. This requires a different decomposition for the two flux components. For the first component, $f$, we decompose $T_{j}$ into two triangles, each of which has an edge parallel to the $x$-axis. For the second component of the flux we split $T_{j}$ into two other triangles, each of which has an edge parallel to the $y$-axis.

We denote the vertices of $T_{j}$ as $V_{j k}, k=1,2,3$, and define the edges of $T_{j}$ as $E_{j 1}=V_{j 2}-V_{j 1}, E_{j 2}=V_{j 3}-V_{j 2}$, and $E_{j 3}=V_{j 1}-V_{j 3}$. The midpoint of the $k$ th edge of $T_{j}$ is $M_{j k}$. I $(a, b)$ denotes the closed interval with endpoints $a$ and $b$. We also use $V_{j k, x}$ (or $V_{j k, y}$ ) to denote the $x$ (or $y$ ) component of $V_{j k}$. To simplify the treatment, we classify the triangles into two categories as portrayed in Figure 3.2.

(i) Type 1: one vertex $V_{j 1}$ bisects the opposite edge in the $y$ direction: the $y$-component $V_{j 1, y} \in I\left(V_{j 2, y}, V_{j 3, y}\right)$ and a different vertex $V_{j 2}$ bisects the opposite edge in the $x$-direction: $V_{j 2, x} \in I\left(V_{j 1, x}, V_{j 3, x}\right)$. (See Figure 3.2 (left).)

(ii) Type 2: the same vertex $V_{j 1}$ bisects the opposite edge in both the $x$ and $y$ directions: $V_{j 1, y} \in I\left(V_{j 2, y}, V_{j 3, y}\right)$ and $V_{j 1, x} \in I\left(V_{j 2, x}, V_{j 3, x}\right)$. (See Figure 3.2 (right).)

Note that these definitions specify our vertex numbering convention. The decomposition for the $g$-flux will depend on the type of triangle.

3.2.1. The decomposition in the $x$-direction. For the flux in the $x$-direction, we decompose $T_{j}$ into two triangles $T_{j}^{x, 1}$ and $T_{j}^{x, 2}$ (see Figure 3.3). Define $V_{j 3}^{x, 1}$ to be the intersection of edge $E_{j 2}$ with the line $y=V_{j 1, y}$. The vertices of $T_{j}^{x, 1}$ are $V_{j 1}^{x, 1}:=V_{j 1}, V_{j 2}^{x, 1}:=V_{j 2}$, and $V_{j 3}^{x, 1}$. The vertices of $T_{j}^{x, 2}$ are $V_{j 1}^{x, 2}:=V_{j 3}^{x, 1}$, $V_{j 2}^{x, 2}:=V_{j 3}$, and $V_{j 3}^{x, 2}:=V_{j 1}$. For both triangles $T_{j}^{x, i}$, the lengths of the sides are $h_{j k}^{x, i}$, the corresponding midpoints are $M_{j k}^{x, i}$, the interior and exterior speeds of propagation are $a_{j k}^{\text {in,out }, x, i}$, and the normals are $n_{j k}^{x, i}=\left(n_{j k, x}^{x, i}, n_{j k, y}^{x, i}\right)$. These definitions imply $M_{j 1}^{x, 1}=M_{j 1}, M_{j 2}^{x, 2}=M_{j 3}, h_{j 1}^{x, 1}=h_{j 1}, h_{j 2}^{x, 2}=h_{j 3}, h_{j 2}^{x, 1}+h_{j 1}^{x, 2}=h_{j 2}, n_{j 1}^{x, 1}=$ 


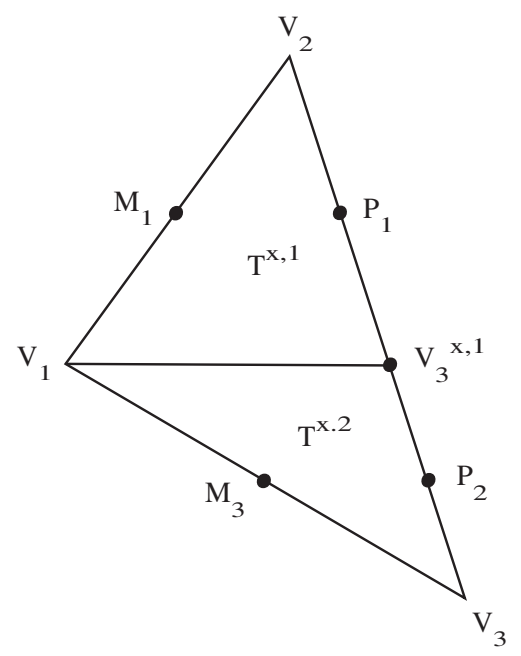

FIG. 3.3. The triangle decomposition for the f-flux.

$n_{j 1, x}, n_{j 2}^{x, 1}=n_{j 1}^{x, 2}=n_{j 2, x}, n_{j 2}^{x, 2}=n_{j 3, x}$, and $n_{j 3, x}^{x, 1}=n_{j 3, x}^{x, 2}=0$. For the velocities we have $a_{j 1}^{\text {in,out }, x, 1}=a_{j 1}^{\text {in,out }}, a_{j 2}^{\text {in, out }, x, 1}=a_{j 2}^{\text {in, out }}, a_{j 1}^{\text {in, out }, x, 2}=a_{j 2}^{\text {in, out }}$, and $a_{j 2}^{\text {in, out }, x, 2}=$ $a_{j 3}^{\text {in,out }}$. The velocity $a_{j 3}^{\text {in,out }, x, i}$ does not play any role in the following computation since $n_{j 3, x}^{x, 1}=n_{j 3, x}^{x, 2}=0$. Finally, the external reconstructions are denoted as $u_{j 1}^{x, 1}=u_{j 1}$, $u_{j 2}^{x, 1}=u_{j 1}^{x, 2}=u_{j 2}$, and $u_{j 2}^{x, 2}=u_{j 3}$.

On each triangle $T_{j}^{x, i}$ we denote the cell average of the component of the flux in the $x$-direction by $\Phi_{j}^{i}$. It is given by

$$
\Phi_{j}^{i}=-\frac{1}{\left|T_{j}^{x, i}\right|} \sum_{k=1}^{2} \frac{h_{j k}^{x, i} n_{j k, x}^{x, i}}{a_{j k}^{\mathrm{in}, x, i}+a_{j k}^{\text {out }, x, i}}\left[a_{j k}^{\mathrm{in}, x, i} f\left(u_{j k}^{x, i}\left(M_{j k}^{x, i}\right)\right)+a_{j k}^{\text {out }, x, i} f\left(u_{j}\left(M_{j k}^{x, i}\right)\right)\right]
$$

for $i=1,2$. The cell average of the $x$-component of the flux over the entire cell $T_{j}$ is taken to be the weighted average

$$
\Phi_{j}:=\frac{\left|T_{j}^{x, 1}\right|}{\left|T_{j}\right|} \Phi_{j}^{1}+\frac{\left|T_{j}^{x, 2}\right|}{\left|T_{j}\right|} \Phi_{j}^{2} .
$$

Since $\sum_{k=1}^{3} h_{j k} n_{j k}=0$ we have $h_{j 1}^{x, 1} n_{j 1, x}^{x, 1}=-h_{j 2}^{x, 1} n_{j 2, x}^{x, 1}$ and $h_{j 2}^{x, 2} n_{j 2, x}^{x, 2}=-h_{j 1}^{x, 2} n_{j 1, x}^{x, 2}$. Hence, $\Phi_{j}$ can be rewritten as

$$
\begin{aligned}
\Phi_{j}= & -\frac{h_{j 1} n_{j 1, x}}{\left|T_{j}\right|}\left[\frac{a_{j 1}^{\text {in }} f_{j 1}^{\text {out }}+a_{j 1}^{\text {out }} f_{j 1}^{\text {in }}}{a_{j 1}^{\text {in }}+a_{j 1}^{\text {out }}}-\frac{a_{j 2}^{\text {in }} f\left(u_{j 2}\left(P_{j 1}\right)\right)+a_{j 2}^{\text {out }} f\left(u_{j}\left(P_{j 1}\right)\right)}{a_{j 2}^{\text {in }}+a_{j 2}^{\text {out }}}\right] \\
& -\frac{h_{j 3} n_{j 3, x}}{\left|T_{j}\right|}\left[\frac{a_{j 3}^{\text {in }} f_{j 3}^{\text {out }}+a_{j 3}^{\text {out }} f_{j 3}^{\text {in }}}{a_{j 3}^{\text {in }}+a_{j 3}^{\text {out }}}-\frac{a_{j 2}^{\text {in }} f\left(u_{j 2}\left(P_{j 2}\right)\right)+a_{j 2}^{\text {out }} f\left(u_{j}\left(P_{j 2}\right)\right)}{a_{j 2}^{\text {in }}+a_{j 2}^{\text {out }}}\right],
\end{aligned}
$$

where $P_{j 1}:=M_{j 2}^{x, 1}$ and $P_{j 2}:=M_{j 1}^{x, 2}$.

Remark. We would like to note that the flux term (3.15) can be derived directly from (2.6) by changing the quadrature points on $E_{j 2}$ to $P_{1}$ and $P_{2}$. 

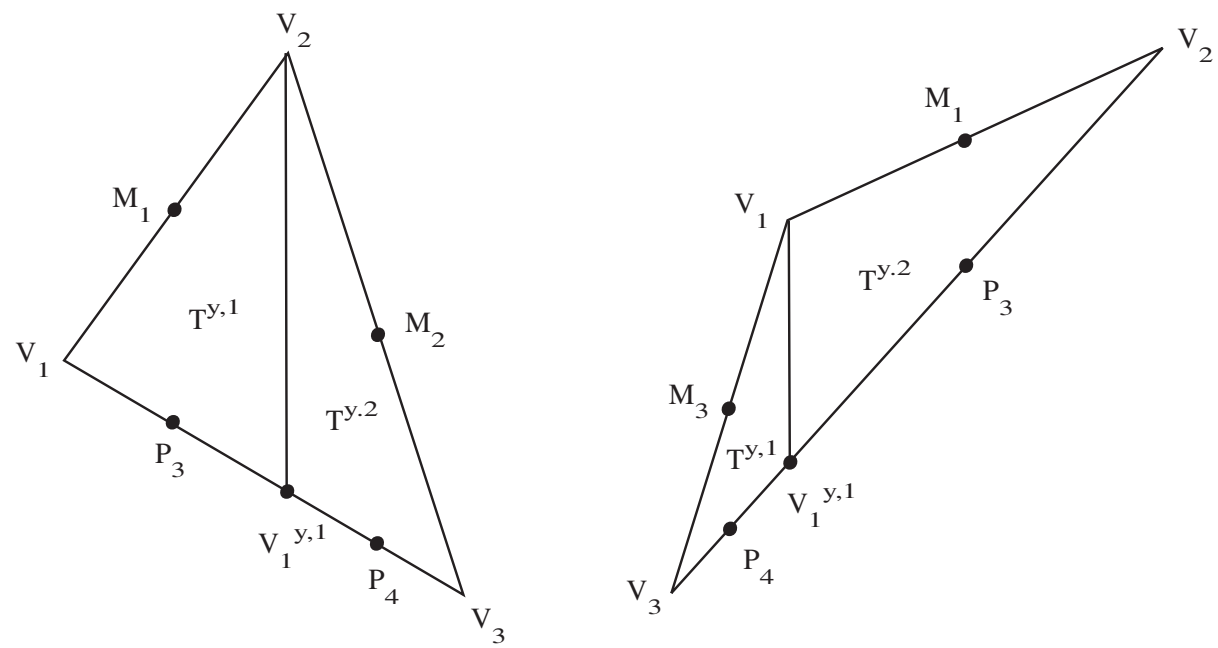

FIG. 3.4. The triangle decomposition for the g-flux. Left: type 1. Right: type 2.

\subsubsection{The decomposition in the $y$-direction.}

Type 1 triangles. We decompose $T_{j}$ into two triangles $T_{j}^{y, 1}$ and $T_{j}^{y, 2}$ (see Figure 3.4 (left)). The intersection of the edge $E_{j 3}$ with the line $x=V_{j 2, x}$ is denoted by $V_{j 1}^{y, 1}$. The vertices of $T_{j}^{y, 1}$ are $V_{j 1}^{y, 1}, V_{j 2}^{y, 1}:=V_{j 1}$, and $V_{j 3}^{y, 1}:=V_{j 2}$. The vertices of $T_{j}^{y, 2}$ are $V_{j 1}^{y, 2}:=V_{j 2}, V_{j 2}^{y, 2}:=V_{j 3}$, and $V_{j 3}^{y, 2}:=V_{j 1}^{y, 1}$. As before, we have $M_{j 2}^{y, 1}=$ $M_{j 1}, M_{j 1}^{y, 2}=M_{j 2}, h_{j 2}^{y, 1}=h_{j 1}, h_{j 1}^{y, 2}=h_{j 2}, h_{j 1}^{y, 1}+h_{j 2}^{y, 2}=h_{j 3}$. The normals are $n_{j 2}^{y, 1}=n_{j 1}, n_{j 1}^{y, 1}=n_{j 2}^{y, 2}=n_{j 3}, n_{j 1}^{y, 2}=n_{j 2}, n_{j 3, y}^{y, 1}=n_{j 3, y}^{y, 2}=0$ and the velocities are $a_{j 1}^{\text {in, out }, y, 1}=a_{j 3}^{\text {in,out }}, a_{j 2}^{\text {in, out, } y, 1}=a_{j 1}^{\text {in, out }}, a_{j 1}^{\text {in,out }, y, 2}=a_{j 2}^{\text {in, out }}, a_{j 2}^{\text {in,out }, y, 2}=a_{j 3}^{\text {in, out }}$. The velocity $a_{j 3}^{\text {in,out }, y, i}$ does not appear in the result because $n_{j 3, y}^{y, 1}=n_{j 3, y}^{y, 2}=0$. Finally, the external reconstructions are $u_{j 1}^{y, 1}=u_{j 3}, u_{j 2}^{y, 1}=u_{j 1}, u_{j 1}^{y, 2}=u_{j 2}$, and $u_{j 2}^{y, 2}=u_{j 3}$.

We denote the cell averages of the component of the flux in the $y$-direction on each triangle $T_{j}^{y, i}$ by $\Gamma_{j}^{i}$. It is given by

$$
\Gamma_{j}^{i}:=-\frac{1}{\left|T_{j}^{y, i}\right|} \sum_{k=1}^{2} \frac{h_{j k}^{y, i} n_{j k, y}^{y, i}}{a_{j k}^{\text {in }, y, i}+a_{j k}^{\text {out }, y, i}}\left[a_{j k}^{\text {in }, y, i} g\left(u_{j k}^{y, i}\left(M_{j k}^{y, i}\right)\right)+a_{j k}^{\text {out }, y, i} g\left(u_{j}\left(M_{j k}^{y, i}\right)\right)\right]
$$

for $i=1,2$. The cell average of the $y$-component of the flux over the entire cell $T_{j}$ is then given by the weighted average

$$
\Gamma_{j}:=\frac{\left|T_{j}^{y, 1}\right|}{\left|T_{j}\right|} \Gamma_{j}^{1}+\frac{\left|T_{j}^{y, 2}\right|}{\left|T_{j}\right|} \Gamma_{j}^{2} .
$$

Clearly $h_{j 2}^{y, 1} n_{j 2, y}^{y, 1}=-h_{j 1}^{y, 1} n_{j 1, y}^{y, 1}$ and $h_{j 1}^{y, 2} n_{j 1, y}^{y, 2}=-h_{j 2}^{y, 2} n_{j 2, y}^{y, 2}$. Therefore $\Gamma_{j}$ can be rewritten as

$$
\begin{aligned}
\Gamma_{j}= & -\frac{h_{j 1} n_{j 1, y}}{\left|T_{j}\right|}\left[\frac{a_{j 1}^{\text {in }} g_{j 1}^{\text {out }}+a_{j 1}^{\text {out }} g_{j 1}^{\text {in }}}{a_{j 1}^{\text {in }}+a_{j 1}^{\text {out }}}-\frac{a_{j 3}^{\text {in }} g\left(u_{j 3}\left(P_{j 3}\right)\right)+a_{j 3}^{\text {out }} g\left(u_{j}\left(P_{j 3}\right)\right)}{a_{j 3}^{\text {in }}+a_{j 3}^{\text {out }}}\right] \\
& -\frac{h_{j 2} n_{j 2, y}}{\left|T_{j}\right|}\left[\frac{a_{j 2}^{\text {in }} g_{j 2}^{\text {out }}+a_{j 2}^{\text {out }} g_{j 2}^{\text {in }}}{a_{j 2}^{\text {in }}+a_{j 2}^{\text {out }}}-\frac{a_{j 3}^{\text {in }} g\left(u_{j 3}\left(P_{j 4}\right)\right)+a_{j 3}^{\text {out }} g\left(u_{j}\left(P_{j 4}\right)\right)}{a_{j 3}^{\text {in }}+a_{j 3}^{\text {out }}}\right],
\end{aligned}
$$


where $P_{j 3}:=M_{j 1}^{y, 1}$ and $P_{j 4}:=M_{j 2}^{y, 2}$.

Type 2 triangles. This case corresponds to Figure 3.4 (right). Analogous computations to those for type 1 triangles provide the cell average of the $y$-component of the flux over the cell $T_{j}$, which this time is given by

$$
\begin{aligned}
\Gamma_{j}= & -\frac{h_{j 1} n_{j 1, y}}{\left|T_{j}\right|}\left[\frac{a_{j 1}^{\text {in }} g_{j 1}^{\text {out }}+a_{j 1}^{\text {out }} g_{j 1}^{\text {in }}}{a_{j 1}^{\text {in }}+a_{j 1}^{\text {out }}}-\frac{a_{j 2}^{\text {in }} g\left(u_{j 2}\left(P_{j 3}\right)\right)+a_{j 2}^{\text {out }} g\left(u_{j}\left(P_{j 3}\right)\right)}{a_{j 2}^{\text {in }}+a_{j 2}^{\text {out }}}\right] \\
& -\frac{h_{j 3} n_{j 3, y}}{\left|T_{j}\right|}\left[\frac{a_{j 3}^{\text {in }} g_{j 3}^{\text {out }}+a_{j 3}^{\text {out }} g_{j 3}^{\text {in }}}{a_{j 3}^{\text {in }}+a_{j 3}^{\text {out }}}-\frac{a_{j 2}^{\text {in }} g\left(u_{j 2}\left(P_{j 4}\right)\right)+a_{j 2}^{\text {out }} g\left(u_{j}\left(P_{j 4}\right)\right)}{a_{j 2}^{\text {in }}+a_{j 2}^{\text {out }}}\right]
\end{aligned}
$$

where $P_{j 3}$ and $P_{j 4}$ are given in Figure 3.4 (right).

3.2.3. The new method (for conservation laws). We would now like to combine all the different ingredients that we developed in the previous section into one scheme. The scheme that we write here is still a scheme for approximating solutions of the conservation law (2.2) without the source term. Based on our preliminary analysis of section 3.1 we know that we will be able to find an admissible discretization of the source terms that will result with a well-balanced scheme. We will treat the source terms in the next section.

We write two versions of the scheme based on the type of triangle. For type 1 triangles, the discretization of the component of the flux in the $x$-direction, $\Phi_{j}$ is given by (3.15) while the discretization of the component of the flux in the $y$-direction, $\Gamma_{j}$, is given by (3.16). In this case the scheme takes the form

$$
\begin{aligned}
\frac{d \bar{u}_{j}}{d t}= & -\frac{h_{j 1} n_{j 1, x}}{\left|T_{j}\right|}\left[\frac{a_{j 1}^{\text {in }} f_{j 1}^{\text {out }}+a_{j 1}^{\text {out }} f_{j 1}^{\text {in }}}{a_{j 1}^{\text {in }}+a_{j 1}^{\text {out }}}-\frac{a_{j 2}^{\text {in }} f\left(u_{j 2}\left(P_{j 1}\right)\right)+a_{j 2}^{\text {out }} f\left(u_{j}\left(P_{j 1}\right)\right)}{a_{j 2}^{\text {in }}+a_{j 2}^{\text {out }}}\right] \\
& -\frac{h_{j 3} n_{j 3, x}}{\left|T_{j}\right|}\left[\frac{a_{j 3}^{\text {in }} f_{j 3}^{\text {out }}+a_{j 3}^{\text {out }} f_{j 3}^{\text {in }}}{a_{j 3}^{\text {in }}+a_{j 3}^{\text {out }}}-\frac{a_{j 2}^{\text {in }} f\left(u_{j 2}\left(P_{j 2}\right)\right)+a_{j 2}^{\text {out }} f\left(u_{j}\left(P_{j 2}\right)\right)}{a_{j 2}^{\text {in }}+a_{j 2}^{\text {out }}}\right] \\
& -\frac{h_{j 1} n_{j 1, y}}{\left|T_{j}\right|}\left[\frac{a_{j 1}^{\text {in }} g_{j 1}^{\text {out }}+a_{j 1}^{\text {out }} g_{j 1}^{\text {in }}}{a_{j 1}^{\text {in }}+a_{j 1}^{\text {out }}}-\frac{a_{j 3}^{\text {in }} g\left(u_{j 3}\left(P_{j 3}\right)\right)+a_{j 3}^{\text {out }} g\left(u_{j}\left(P_{j 3}\right)\right)}{a_{j 3}^{\text {in }}+a_{j 3}^{\text {out }}}\right] \\
& -\frac{h_{j 2} n_{j 2, y}}{\left|T_{j}\right|}\left[\frac{a_{j 2}^{\text {in }} g_{j 2}^{\text {out }}+a_{j 2}^{\text {out }} g_{j 2}^{\text {in }}}{a_{j 2}^{\text {in }}+a_{j 2}^{\text {out }}}-\frac{a_{j 3}^{\text {in }} g\left(u_{j 3}\left(P_{j 4}\right)\right)+a_{j 3}^{\text {in }} g\left(u_{j}\left(P_{j 4}\right)\right)}{a_{j 3}^{\text {in }}+a_{j 3}^{\text {out }}}\right] \\
& +\frac{1}{\left|T_{j}\right|} \sum_{k=1}^{3} h_{j k} \frac{a_{j k}^{\text {in }} a_{j k}^{\text {out }}}{a_{j k}^{\text {in }}+a_{j k}^{\text {out }}}\left[u_{j k}^{\text {out }}-u_{j k}^{\text {in }}\right] .
\end{aligned}
$$

Here $P_{1}=V_{2}-\frac{1}{2} \frac{E_{1, y}}{E_{2, y}} E_{2}, P_{2}=V_{3}+\frac{1}{2} \frac{E_{3, y}}{E_{2, y}} E_{2}, P_{3}=V_{1}+\frac{1}{2} \frac{E_{1, x}}{E_{3, x}} E_{3}$, and $P_{4}=V_{3}-$ $\frac{1}{2} \frac{E_{2, x}}{E_{3, x}} E_{3}$.

For type 2 triangles we replace the discretization of $\Gamma_{j}$ by the one given in (3.17) 
ending with

$$
\begin{aligned}
\frac{d \bar{u}_{j}}{d t}= & -\frac{h_{j 1} n_{j 1, x}}{\left|T_{j}\right|}\left[\frac{a_{j 1}^{\text {in }} f_{j 1}^{\text {out }}+a_{j 1}^{\text {out }} f_{j 1}^{\text {in }}}{a_{j 1}^{\text {in }}+a_{j 1}^{\text {out }}}-\frac{a_{j 2}^{\text {in }} f\left(u_{j 2}\left(P_{j 1}\right)\right)+a_{j 2}^{\text {out }} f\left(u_{j}\left(P_{j 1}\right)\right)}{a_{j 2}^{\text {in }}+a_{j 2}^{\text {out }}}\right] \\
& -\frac{h_{j 3} n_{j 3, x}}{\left|T_{j}\right|}\left[\frac{a_{j 3}^{\text {in }} f_{j 3}^{\text {out }}+a_{j 3}^{\text {out }} f_{j 3}^{\text {in }}}{a_{j 3}^{\text {in }}+a_{j 3}^{\text {out }}}-\frac{a_{j 2}^{\text {in }} f\left(u_{j 2}\left(P_{j 2}\right)\right)+a_{j 2}^{\text {out }} f\left(u_{j}\left(P_{j 2}\right)\right)}{a_{j 2}^{\text {in }}+a_{j 2}^{\text {out }}}\right] \\
& -\frac{h_{j 1} n_{j 1, y}}{\left|T_{j}\right|}\left[\frac{a_{j 1}^{\text {in }} g_{j 1}^{\text {out }}+a_{j 1}^{\text {out }} g_{j 1}^{\text {in }}}{a_{j 1}^{\text {in }}+a_{j 1}^{\text {out }}}-\frac{a_{j 2}^{\text {in }} g\left(u_{j 2}\left(P_{j 3}\right)\right)+a_{j 2}^{\text {out }} g\left(u_{j}\left(P_{j 3}\right)\right)}{a_{j 2}^{\text {in }}+a_{j 2}^{\text {out }}}\right] \\
& -\frac{h_{j 3} n_{j 3, y}}{\left|T_{j}\right|}\left[\frac{a_{j 2}^{\text {in }} g_{j 3}^{\text {out }}+a_{j 2}^{\text {out }} g_{j 3}^{\text {in }}}{a_{j 3}^{\text {in }}+a_{j 3}^{\text {out }}}-\frac{a_{j 2}^{\text {in }} g\left(u_{j 2}\left(P_{j 4}\right)\right)+a_{j 2}^{\text {in }} g\left(u_{j}\left(P_{j 4}\right)\right)}{a_{j 2}^{\text {in }}+a_{j 2}^{\text {out }}}\right] \\
& +\frac{1}{\left|T_{j}\right|} \sum_{k=1}^{3} h_{j k} \frac{a_{j k}^{\text {in }} a_{j k}^{\text {out }}}{a_{j k}^{\text {in }}+a_{j k}^{\text {out }}}\left[u_{j k}^{\text {out }}-u_{j k}^{\text {in }}\right] .
\end{aligned}
$$

In this case $P_{1}=V_{2}-\frac{1}{2} \frac{E_{1, y}}{E_{2, y}} E_{2}, P_{2}=V_{3}+\frac{1}{2} \frac{E_{3, y}}{E_{2, y}} E_{2}, P_{3}=V_{2}-\frac{1}{2} \frac{E_{1, x}}{E_{2, x}} E_{2}$, and $P_{4}=V_{3}+\frac{1}{2} \frac{E_{3, x}}{E_{2, x}} E_{2}$.

Remarks.

1. For a general triangulation our method will not be conservative because the fluxes from adjacent triangles will not in general be evaluated at the same point on the edge. Our tests with irregular triangulations in section 4 show, however, that this failure of conservation does not lead to serious problems.

2. A simple case of interest is that of coordinate-aligned right triangles. Such triangles can be considered to be of type 1 with $n_{j 2, y}=n_{j 3, x}=0$ and $P_{j 1}=M_{j 2}$ and $P_{j 3}=M_{j 3}$, which means that the method becomes (taking into account that $\left.\sum_{k=1}^{3} h_{j k} n_{j k}=0\right)$

$$
\begin{aligned}
\frac{d \bar{u}_{j}}{d t}= & -\frac{1}{\left|T_{j}\right|}\left[\frac{a_{j 1}^{\text {in }} f_{j 1}^{\text {out }}+a_{j 1}^{\text {out }} f_{j 1}^{\text {in }}}{a_{j 1}^{\text {in }}+a_{j 1}^{\text {out }}} h_{j 1} n_{j 1, x}+\frac{a_{j 2}^{\text {in }} f_{j 2}^{\text {out }}+a_{j 2}^{\text {out }} f_{j 1}^{\text {in }}}{a_{j 2}^{\text {in }}+a_{j 2}^{\text {out }}} h_{j 2} n_{j 2, x}\right] \\
& -\frac{1}{\left|T_{j}\right|}\left[\frac{a_{j 1}^{\text {in }} g_{j 1}^{\text {out }}+a_{j 1}^{\text {out }} g_{j 1}^{\text {in }}}{a_{j 1}^{\text {in }}+a_{j 1}^{\text {out }}} h_{j 1} n_{j 1, y}+\frac{a_{j 3}^{\text {in }} g_{j 3}^{\text {out }}+a_{j 3}^{\text {out }} g_{j 1}^{\text {in }}}{a_{j 3}^{\text {in }}+a_{j 3}^{\text {out }}} h_{j 3} n_{j 3, y}\right] \\
& +\frac{1}{\left|T_{j}\right|} \sum_{k=1}^{3} h_{j k} \frac{a_{j k}^{\text {in }} a_{j k}^{\text {out }}}{a_{j k}^{\text {in }}+a_{j k}^{\text {out }}}\left[u_{j k}^{\text {out }}-u_{j k}^{\text {in }}\right] .
\end{aligned}
$$

As expected, in this case the method (3.20) coincides with the KP method (2.7).

3.2.4. Adding the source term. We return to the shallow water equations (1.1). Our goal is now to find an admissible discretization of the cell average of the source terms that will preserve stationary steady states. Such a term will be added to the RHS of (3.18) or (3.19) depending on the type of the triangle. In the following we assume type 1 triangles. Similar analysis holds for type 2 triangles. We also note that the first equation in the system (1.1) is homogeneous. This means that we only have to consider the remaining two equations when dealing with the source terms. We recall from section 3.1 that in stationary steady states all the velocities are equal, $a_{j k}^{\text {in }}=a_{j k}^{\text {out }}=a_{j k}$. Our new method (3.18) for the last two equations of (1.1) then 
becomes

$$
\begin{aligned}
0= & -\frac{h_{j 1} n_{j 1, x}}{2\left|T_{j}\right|}\left[\left(\begin{array}{c}
\left(w\left(M_{j 1}\right)-B\left(M_{j 1}\right)\right)^{2} \\
0
\end{array}\right)-\left(\begin{array}{c}
\left(w\left(P_{j 1}\right)-B\left(P_{j 1}\right)\right)^{2} \\
0
\end{array}\right)\right] \\
& -\frac{h_{j 3} n_{j 3, x}}{2\left|T_{j}\right|}\left[\left(\begin{array}{c}
\left(w\left(M_{j 3}\right)-B\left(M_{j 3}\right)\right)^{2} \\
0
\end{array}\right)-\left(\begin{array}{c}
\left(w\left(P_{j 2}\right)-B\left(P_{j 2}\right)\right)^{2} \\
0
\end{array}\right)\right] \\
& -\frac{h_{j 1} n_{j 1, y}}{2\left|T_{j}\right|}\left[\left(\begin{array}{c}
\left(w\left(M_{j 1}\right)-B\left(M_{j 1}\right)\right)^{2}
\end{array}\right)-\left(\begin{array}{c}
0 \\
\left(w\left(P_{j 3}\right)-B\left(P_{j 3}\right)\right)^{2}
\end{array}\right)\right] \\
& -\frac{h_{j 2} n_{j 2, y}}{2\left|T_{j}\right|}\left[\left(\begin{array}{c}
0 \\
\left(w\left(M_{j 2}\right)-B\left(M_{j 2}\right)\right)^{2}
\end{array}\right)-\left(\begin{array}{c}
\left(w\left(P_{j 4}\right)-B\left(P_{j 4}\right)\right)^{2}
\end{array}\right)\right]+\left(\begin{array}{c}
\bar{S}_{j}^{1} \\
\bar{S}_{j}^{2}
\end{array}\right) .
\end{aligned}
$$

The last term in (3.21) represents the average of the source, i.e., $\bar{S}_{j}^{1}=\operatorname{avg}(-(w-$ $\left.B) B_{x}\right)$ and $\bar{S}_{j}^{2}=\operatorname{avg}\left(-(w-B) B_{y}\right)$, where both averages are taken over $T_{j}$.

We use (3.21) to determine the admissible discretizations of the cell averages of the source terms. For constant $w$ the source terms (given by (3.21)) can be rewritten as

$$
\begin{aligned}
\bar{S}_{j}^{1}= & -\frac{h_{j 1} n_{j 1, x}}{2\left|T_{j}\right|}\left[w_{M_{j 1}}-B_{M_{j 1}}+w_{P_{j 1}}-B_{P_{j 1}}\right]\left[B_{M_{j 1}}-B_{P_{j 1}}\right] \\
& -\frac{h_{j 3} n_{j 3, x}}{2\left|T_{j}\right|}\left[w_{M_{j 3}}-B_{M_{j 3}}+w_{P_{j 2}}-B_{P_{j 2}}\right]\left[B_{M_{j 3}}-B_{P_{j 2}}\right], \\
\bar{S}_{j}^{2}= & -\frac{h_{j 1} n_{j 1, y}}{2\left|T_{j}\right|}\left[w_{M_{j 1}}-B_{M_{j 1}}+w_{P_{j 3}}-B_{P_{j 3}}\right]\left[B_{M_{j 1}}-B_{P_{j 3}}\right] \\
& -\frac{h_{j 2} n_{j 2, y}}{2\left|T_{j}\right|}\left[w_{M_{j 2}}-B_{M_{j 2}}+w_{P_{j 4}}-B_{P_{j 4}}\right]\left[B_{M_{j 2}}-B_{P_{j 4}}\right] .
\end{aligned}
$$

Here, we use the notation $w_{M_{1}}:=w\left(M_{j 1}\right)$, etc. We take the expressions in (3.22) as the discretization of the source even when $w$ is not constant. Similar expressions can be easily written for type 2 triangles.

LEMma 3.1. The source discretizations $\bar{S}_{j}^{i}$ given by (3.22) are consistent approximations of the source terms in (1.1) and lead to detailed balance in the stationary steady-state case (3.21).

Before proving Lemma 3.1 we consider the following geometrical lemma.

Lemma 3.2. With $M$ and $P$ defined as in section 3.2 .1 (suppressing $j$ ),

$$
M_{1, x}-P_{1, x}=-\frac{|T|}{h_{2} n_{2, x}}, \quad M_{3, x}-P_{2, x}=-\frac{|T|}{h_{2} n_{2, x}} .
$$

Proof. $M_{1}=\frac{1}{2}\left(V_{1}+V_{2}\right)$, and for some $s, P_{1}=V_{2}+s\left(V_{3}-V_{2}\right)$. We require that $P_{1, y}=M_{1, y}$, which implies that

$$
s=\frac{1}{2} \frac{V_{1, y}-V_{2, y}}{V_{3, y}-V_{2, y}}
$$

Therefore

$$
M_{1, x}-P_{1, x}=\frac{1}{2}\left(V_{1, x}+V_{2, x}\right)-V_{2, x}-\frac{1}{2} \frac{V_{1, y}-V_{2, y}}{V_{3, y}-V_{2, y}}\left(V_{3, x}-V_{2, x}\right)=-\frac{1}{2} \frac{E_{1} \times E_{2}}{E_{2, y}} .
$$


When the orientation of the vertices of $T_{j}$ is clockwise, $E_{1} \times E_{2}=-2|T|$ and $n_{2}=$ $\frac{1}{h_{2}}\left(-E_{2, y}, E_{2, x}\right)$, while when the orientation is counterclockwise, $E_{1} \times E_{2}=2|T|$ and $n_{2}=\frac{1}{h_{2}}\left(E_{2, y},-E_{2, x}\right)$, so

$$
M_{1, x}-P_{1, x}=-\frac{|T|}{h_{2} n_{2, x}} .
$$

Similar arguments hold for $M_{3, x}-P_{2, x}$.

Proof of Lemma 3.1. We show that $\bar{S}_{j}^{1} \approx \operatorname{avg}\left(-(w-B) B_{x}\right)$. To simplify the notations we suppress the index $j$. From Lemma 3.2 we know that $M_{1, x}-P_{1, x}=$ $-\frac{|T|}{h_{2} n_{2, x}}$. Since $M_{1, y}=P_{1, y}$ we have $\frac{B_{M_{1}}-B_{P_{1}}}{M_{1, x}-P_{1, x}}=B_{x}+O\left(\left|M_{1}-P_{1}\right|^{2}\right)$ at the midpoint between $M_{1}$ and $P_{1}$. Hence the first part of $\bar{S}_{j}^{1}$ in (3.22) becomes

$$
\begin{array}{r}
\frac{h_{1} n_{1, x}}{2 h_{2} n_{2, x}}\left(w_{M_{1}}-B_{M_{1}}+w_{P_{1}}-B_{P_{1}}\right) \frac{B_{M_{1}}-B_{P_{1}}}{M_{1, x}-P_{1, x}} \\
\approx \frac{h_{1} n_{1, x}}{2 h_{2} n_{2, x}}\left(w_{M_{1}}-B_{M_{1}}+w_{P_{1}}-B_{P_{1}}\right) B_{x} .
\end{array}
$$

Applying a similar argument to the second term of $\bar{S}_{j}^{1}$ in (3.22) gives

$$
\begin{aligned}
\bar{S}_{j}^{1} \approx \frac{1}{2}( & \frac{h_{1} n_{1, x}}{h_{2} n_{2, x}}\left(w_{M_{1}}-B_{M_{1}}+w_{P_{1}}-B_{P_{1}}\right) \\
& \left.+\frac{h_{3} n_{3, x}}{h_{2} n_{2, x}}\left(w_{M_{3}}-B_{M_{3}}+w_{P_{2}}-B_{P_{2}}\right)\right) B_{x} .
\end{aligned}
$$

Clearly, the coefficient of $B_{x}$ in (3.23) is a discretization of a weighted average of $-(w-B)$. For example, when $w-B$ is constant we have

$$
\bar{S}_{j}^{1} \approx \frac{h_{1} n_{1, x}+h_{3} n_{3, x}}{h_{2} n_{2, x}}(w-B) B_{x}=-(w-B) B_{x} .
$$

Similar arguments hold for $\bar{S}_{j}^{2}$.

Remark. If we consider a Cartesian mesh split into triangles along a diagonal, it is easy to see that our method reduces to the method in [14].

4. Numerical examples. The scheme developed in section 3.2 did not assume any particular reconstruction. There are several different second-order reconstructions on triangular meshes that are being used in the literature (see [16] and the references therein). We briefly describe the one we used in our simulations.

The starting point is the limited least-squares estimate of the gradients as done in [2]. The first step is to compute a least-squares estimate of the gradient of a field $f$ on the triangle $T_{j}, \tilde{\nabla}_{j} f$. We then limit the gradient $\mathcal{D}_{j} f$ component by component as

$$
\mathcal{D}_{j} f=\mathcal{M} \mathcal{M}\left(\tilde{\nabla}_{j} f, \tilde{\nabla}_{j 1} f, \tilde{\nabla}_{j 2} f, \tilde{\nabla}_{j 3} f\right),
$$

where $\tilde{\nabla}_{j k} f$ is the least-squares gradient estimate on $T_{j k}$ and $\mathcal{M} \mathcal{M}$ stands for the usual MinMod limiter

$$
\mathcal{M N M}\left(x_{1}, x_{2}, \ldots\right):= \begin{cases}\min _{j}\left\{x_{j}\right\} & \text { if } x_{j}>0 \quad \forall j \\ \max _{j}\left\{x_{j}\right\} & \text { if } x_{j}<0 \quad \forall j \\ 0 & \text { otherwise }\end{cases}
$$



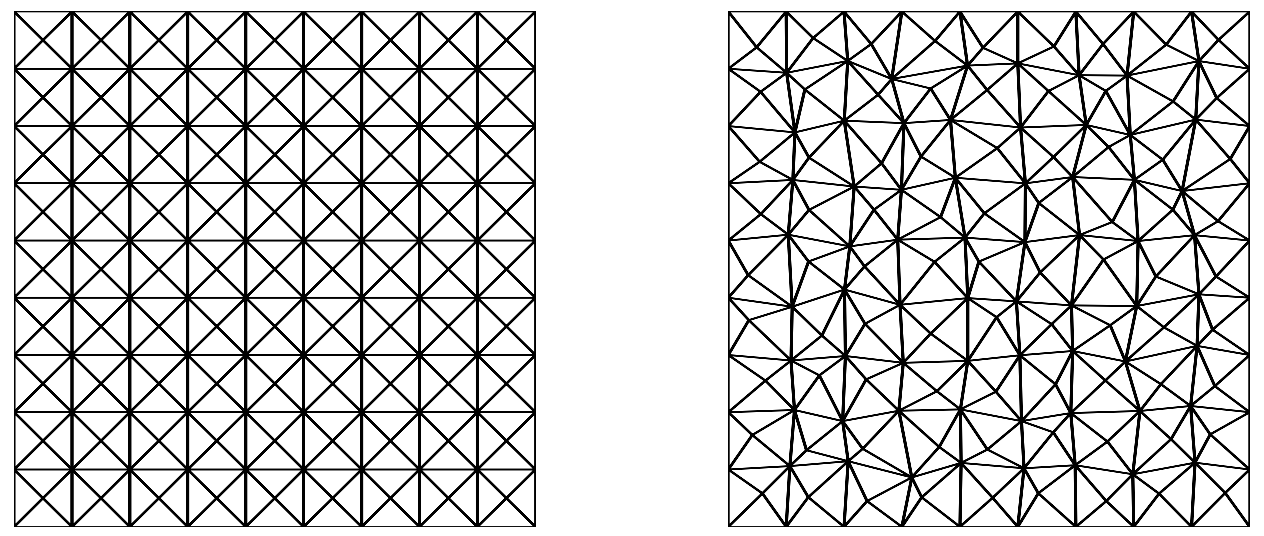

FIG. 4.1. The two types of triangle meshes. Left: Uniform triangulation. Right: Perturbed (nonuniform) triangulation.

We use the gradients $\mathcal{D}_{j}$ to construct a piecewise linear reconstruction for the point values of each triangle edge $E_{j k}$ as

$$
u_{j}(\vec{x})=\bar{u}_{j}+\mathcal{N} \mathcal{N}\left(\mathcal{D}_{j} u \cdot\left(\vec{x}-\vec{x}_{j}\right), \mathcal{D}_{j k} u \cdot\left(\vec{x}-\vec{x}_{j}\right)\right) .
$$

Here $\mathcal{D}_{j k} u$ is the limited gradient estimate on $T_{j k}, \vec{x}_{j}$ is the center of $T_{j}$, and $\vec{x} \in E_{j k}$. We find that this double use of the MinMod limiter minimizes spurious oscillations while preserving the second-order accuracy of the reconstruction.

We use an adaptive time step given by

$$
\Delta t=0.9 \min _{j} \frac{r_{j}}{\left|\lambda_{N}^{j}\right|}
$$

where $r_{j}$ is the radius of $T_{j}$ and $\left|\lambda_{N}^{j}\right|$ is the norm of the largest eigenvalues of the Jacobian of the flux on $T_{j}$.

We use two types of meshes in most of our simulations. The first is a uniform triangulation of a Cartesian mesh with $N \times N$ nodes and constant spacings $\Delta x$ and $\Delta y$, which divides each Cartesian cell into four triangles. To test our method on more general meshes, our second type of mesh is generated from a uniform triangular mesh with a perturbation of the coordinates of the interior vertices. Examples of both meshes are shown in Figure 4.1. The only exception is Example 6, which uses a uniform triangulation of a warped Cartesian mesh.

Example 1: Accuracy tests for a two-dimensional Burgers equation. We test the accuracy of our method (3.18)-(3.19) on nonlinear problems by applying it to the two-dimensional Burgers equation

$$
\left\{\begin{array}{l}
u_{t}+\frac{1}{2}\left(u^{2}\right)_{x}+\frac{1}{2}\left(u^{2}\right)_{y}=0, \quad[-2 \pi, 2 \pi]^{2}, \\
u(x, y, t=0)=\sin \left(\frac{x+y}{2}\right)
\end{array}\right.
$$

with periodic boundary conditions. The relative $L^{1}$-error (i.e., the $L^{1}$-error divided by the $L^{1}$-norm of the exact solution) of our method at $T=0.5$ is shown in Table 4.1 for uniform and perturbed triangulations. The computed cell averages after singularity formation at $T=1.5$ are shown in Figure 4.2. Note the sharp shocks that are captured by our method. 
TABLE 4.1

$L^{1}$-error and convergence rates for Burgers equation (4.1) at $T=0.5$ on a uniform and a perturbed triangulation of an $N \times N$ Cartesian mesh.

\begin{tabular}{|c||c|c||c|c|}
\hline \multicolumn{1}{|c||}{} & \multicolumn{2}{c||}{ Uniform triangulation } & \multicolumn{2}{c|}{ Perturbed triangulation } \\
\hline$N$ & Relative $L^{1}$-error & $L^{1}$-order & Relative $L^{1}$-error & $L^{1}$-order \\
\hline \hline 10 & 0.064 & - & 0.064 & - \\
\hline 20 & 0.014 & 2.20 & 0.014 & 2.20 \\
\hline 40 & $4.68 \times 10^{-3}$ & 1.58 & $4.65 \times 10^{-3}$ & 1.59 \\
\hline 80 & $1.37 \times 10^{-3}$ & 1.77 & $1.36 \times 10^{-3}$ & 1.77 \\
\hline
\end{tabular}
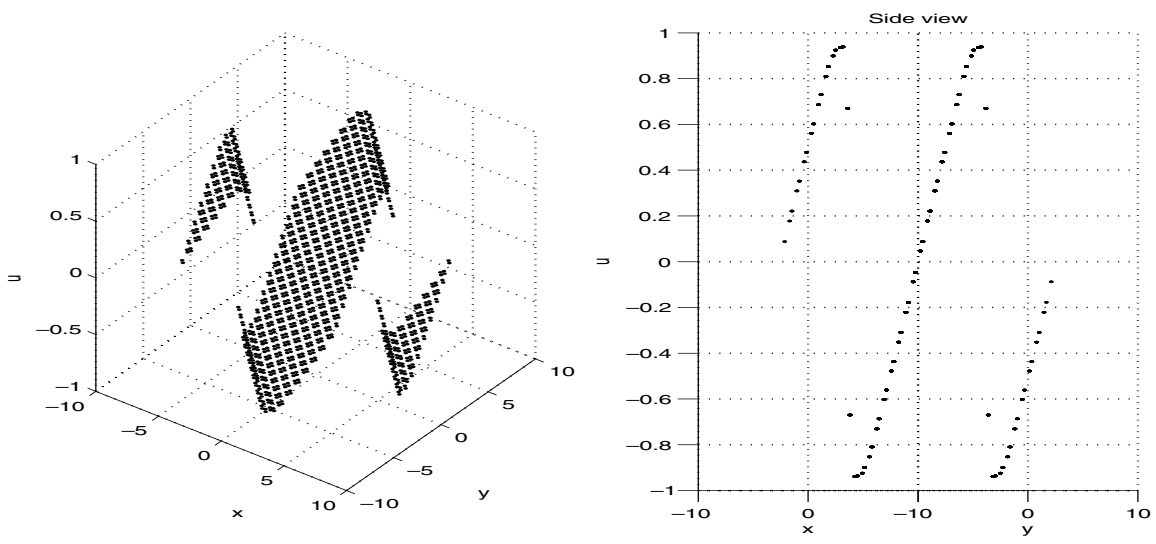

FIG. 4.2. Burgers equation at $T=1.5$ on a uniform triangulation of a $20 \times 20$ Cartesian mesh. Left: oblique view. Right: side view.

Example 2: The Sod problem. To test our method (3.18)-(3.19) on a system of equations we apply it to the Sod problem for the Euler equations

$$
\left(\begin{array}{c}
\rho \\
\rho u \\
\rho v \\
E
\end{array}\right)_{t}+\left(\begin{array}{c}
\rho u \\
\rho u^{2}+p \\
\rho u v \\
u(E+p)
\end{array}\right)_{x}+\left(\begin{array}{c}
\rho v \\
\rho u v \\
\rho v^{2}+p \\
v(E+p)
\end{array}\right)_{y}=0 .
$$

Here $\rho$ is the density, $(u, v)$ is the velocity, and $E$ is the energy. The equation of state for the pressure is $p=(\gamma-1)\left[E-\frac{\rho}{2}\left(u^{2}+v^{2}\right)\right]$. We set $\gamma=1.4$ and take the initial conditions (constant in the $y$-direction)

$$
(p, \rho, u, v)= \begin{cases}(1.0,1.0,0.0,0.0), & x<0.5 \\ (0.1,0.125,0.0,0.0), & x>0.5 .\end{cases}
$$

Figure 4.3 shows the computed cell averages at $T=0.16$ using our method from section 3.2 projected onto the $y=0$ plane. A reference one-dimensional solution is also shown. The two-dimensional problem is run on both a uniform triangulation based on a $300 \times 30$ Cartesian mesh on the domain $[0,1] \times[0,0.1]$ as well as a perturbation of that mesh. The one-dimensional reference solution is computed using the secondorder central method of [15] with 3000 points in the domain $[0,1]$. As expected, the quality of the solution slightly degrades on the nonuniform mesh.

Example 3: A balance test. In this example we demonstrate the well-balanced property of our scheme (3.18)-(3.19) with the source discretization (3.22). 

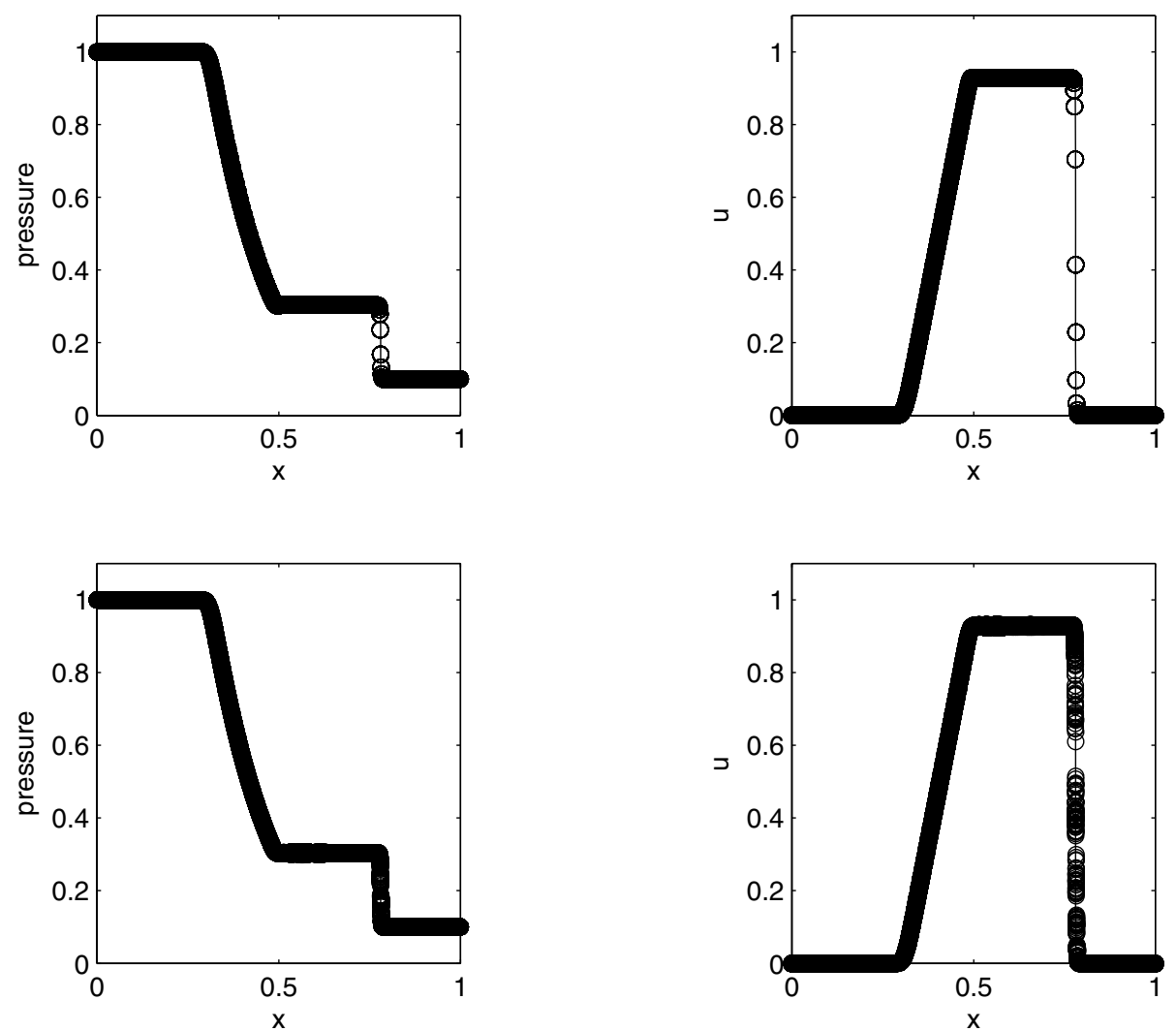

FIG. 4.3. The pressure (left) and u-velocity (right) fields of the Sod problem at $T=0.16$ on a uniform triangulation (top) and a perturbed triangulation (bottom). The circles show an orthogonal projection onto the $y=0$ plane of the solution of the two-dimensional problem on a triangular mesh. The line shows a one-dimensional reference solution computed with the second-order central-upwind method of [15].

As a simple test of balance, we consider the shallow water problem with initial conditions that represent a stationary steady state. We choose $w(x, y, t=0)=2$, $u(x, y, t=0)=v(x, y, t=0)=0$, and a bottom topography given by $B(x, y)=$ $\sin (2 \pi x)+\cos (2 \pi y)$ on the domain $[0,1]^{2}$. We assume periodic boundary conditions. This initial value problem has the trivial stationary steady-state solution $w(x, y, t)=$ $2, u(x, y, t)=v(x, y, t)=0$ for all $t$.

The relative $L^{1}$-errors at $T=1$ on both uniform and perturbed triangulations based on a $10 \times 10$ Cartesian mesh are given in Table 4.2. This table also shows the result found using the KP method [16] with the same source discretization (3.22) which is not balanced for this scheme. We see that our method balances to machine accuracy.

Example 4: Propagating waves with a bottom topography. We apply our method to a test problem from [18] of a small perturbation of a steady-state problem on the domain $[0,2] \times[0,1]$ with periodic boundary conditions in the $y$-direction. The bottom topography is the elliptical Gaussian mound given by

$$
B(x, y)=0.8 \exp \left(-5(x-0.9)^{2}-50(y-0.5)^{2}\right),
$$


TABLE 4.2

The relative $L^{1}$-error in the balance for (3.18), (3.19), (3.22) and for the KP scheme with the source discretization (3.22).

\begin{tabular}{|c||c||c|}
\hline Method & Uniform triangulation & Perturbed triangulation \\
\hline \hline$(3.18)-(3.19)-(3.22)$ & $3.5 \times 10^{-17}$ & $6.9 \times 10^{-19}$ \\
\hline$[16]+(3.22)$ & $3.0 \times 10^{-3}$ & $3.5 \times 10^{-3}$ \\
\hline
\end{tabular}
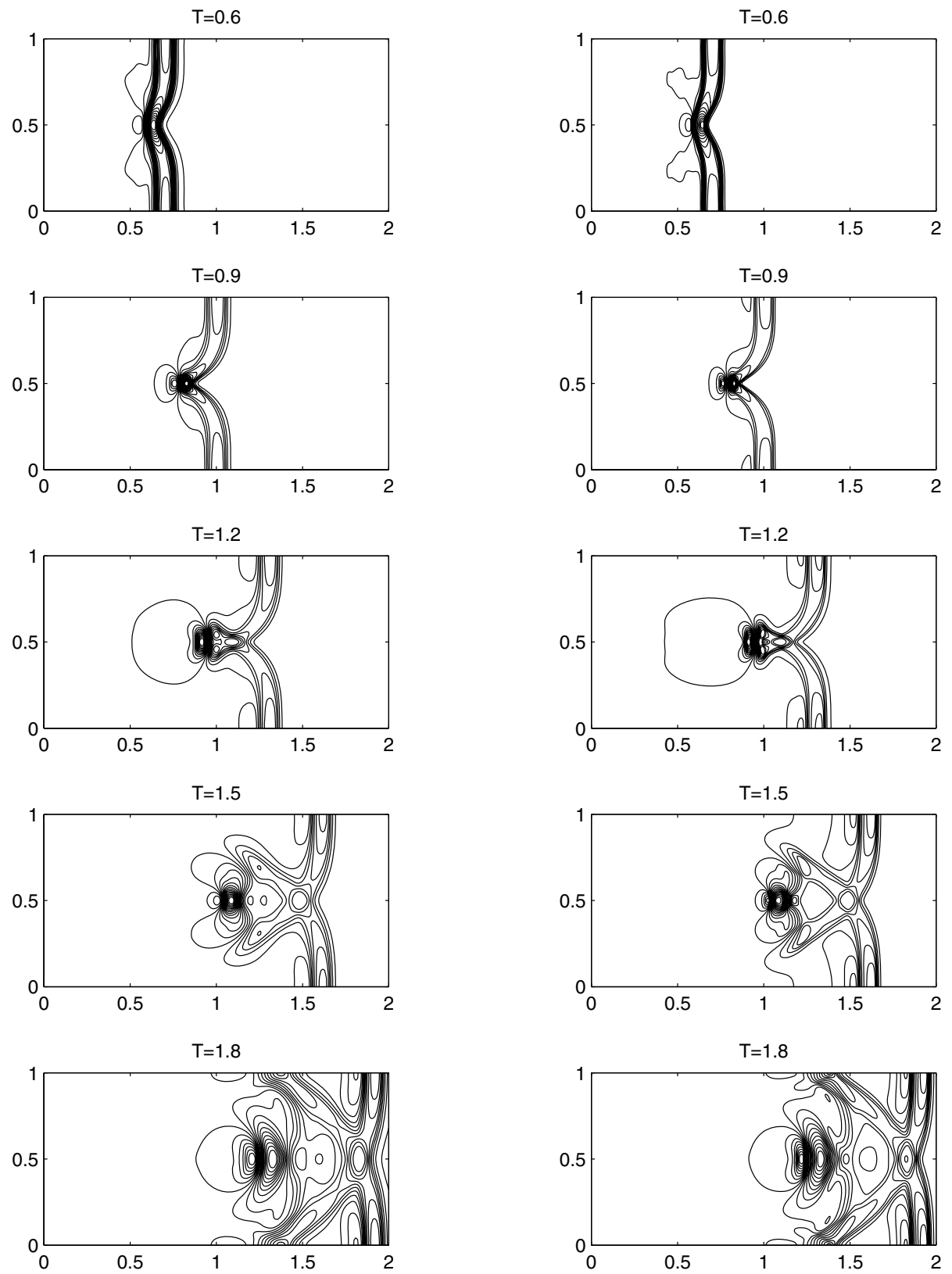

FiG. 4.4. Wave propagation over an elliptical hump at various times. Left: uniform triangulation based on a $200 \times 100$ mesh. Right: uniform triangulation based on a $400 \times 200$ mesh. 
(a)

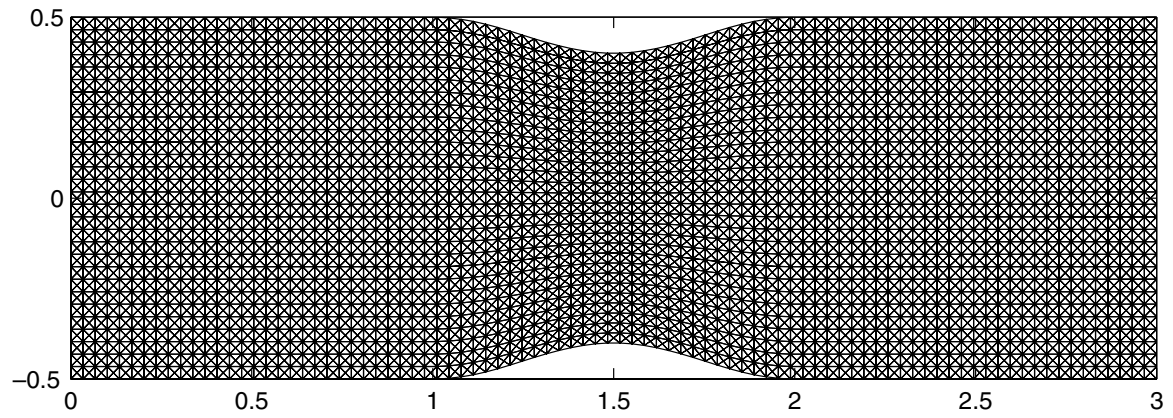

(b)

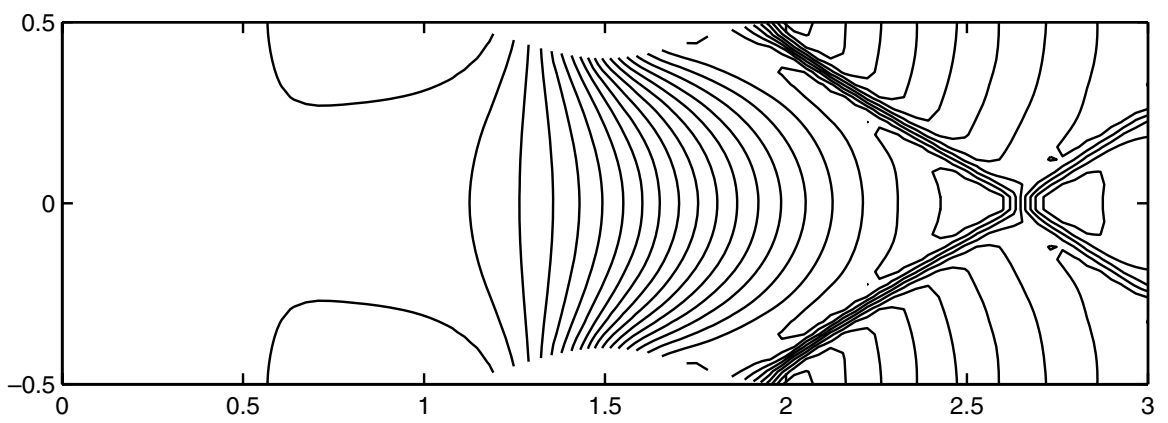

(c)

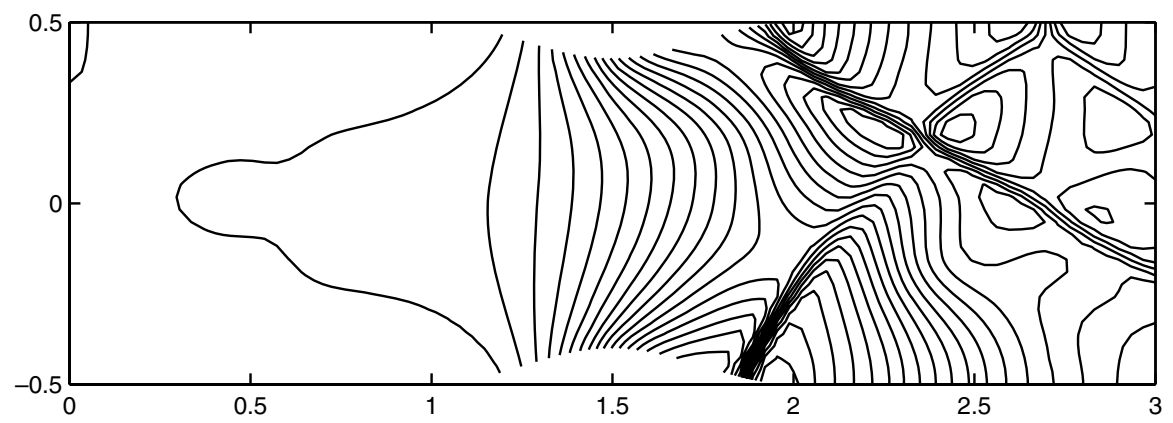

(d)

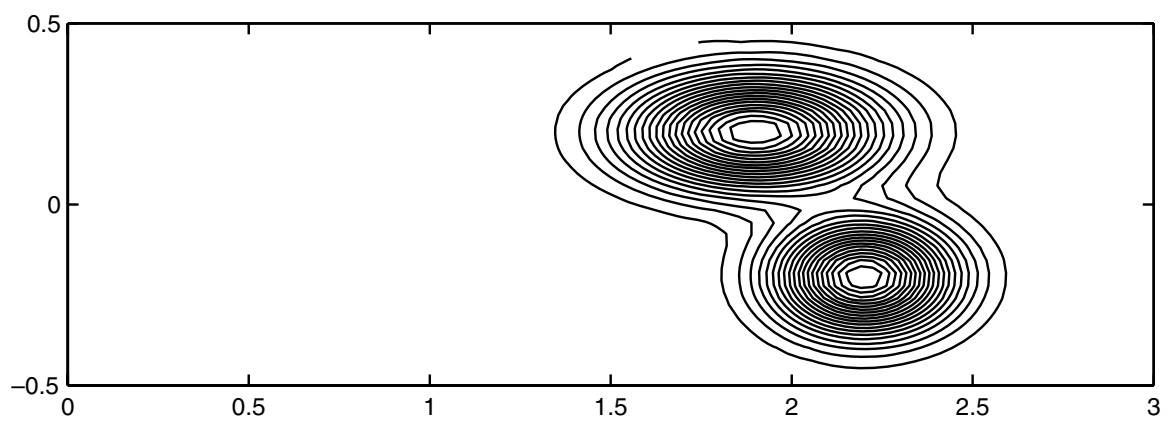

FIG. 4.5. Example 6: (a) The mesh. (b) Contours of $w$ for a critical flow through a convergingdiverging channel with flat bottom at $T=7$. (c) Contours of $w$ for a critical flow through a converging-diverging channel with the topography shown below at $T=7$. (d) The bottom topography for figure (c). 
and the initial conditions are

$$
(w, u, v)= \begin{cases}(1.01,0.0,0.0) & \text { if } 0.05<x<0.15 \\ (1.0,0.0,0.0) & \text { otherwise }\end{cases}
$$

Figure 4.4 shows the result of our method at various times on a uniform triangulation of both a $200 \times 100$ and $400 \times 200$ Cartesian mesh. These results are in good agreement with other methods on Cartesian grids (see $[14,18]$ ).

Example 5: Converging-diverging channel with bottom topography. Our final example is that of a converging-diverging channel with critical flow adapted from [12]. The channel is defined on the domain $[0,3] \times[-0.5,0.5]$ with a half-cosine constriction centered at $x=1.5$. The mesh for this example is shown in Figure 4.5(a). It is a nonuniform triangulation obtained by warping a uniform triangulation of a Cartesian mesh under the mapping $(x, y) \rightarrow\left(x,\left(1-0.2 \cos ^{2}(\pi(x-1.5))\right) y\right)$ when $|x-1.5|<0.5$. The initial data is $w=1, u=v=0$. The $y$-boundaries are reflecting. The left $x$-boundary is an inflow boundary with $u=5.0$ and the right $x$-boundary is a zeroth-order outflow boundary. We run the simulations on a $90 \times 30$ mesh until $T=7$ after the steady state is achieved. We would like to emphasize that this is not a stationary steady state.

We first present this example with a flat bottom, with contours of $w$ shown in Figure 4.5(b). Figure 4.5(c) shows the same channel at the same time with bottom topography

$$
\begin{aligned}
B(x, y)=0.8 & \left(\exp \left(-10(x-1.9)^{2}-50(y-0.2)^{2}\right)\right. \\
& \left.+\exp \left(-20(x-2.2)^{2}-50(y+0.2)^{2}\right)\right) .
\end{aligned}
$$

This topography is shown in Figure 4.5(d) and represents two elliptical Gaussian mounds just downflow from the constriction.

We choose the initial and boundary conditions such that stationary shocks are generated. The symmetry of the flat-bottom case (Figure 4.5(b)) is broken by the nonsymmetric bottom (Figures 4.5(c)-(d)). These results are in qualitative agreement with those that are shown in [12]. We note that the simulations in [12] correspond only to the flat bottom case.

5. Conclusion. In this paper we presented a well-balanced semidiscrete central scheme on triangular meshes for shallow water equations with bottom topography. This scheme is derived by changing the quadrature rules for the flux integrals that were proposed in [16]. A careful discretization of the source term then allows us to preserve stationary steady states.

The numerical flux is complemented by a new second-order reconstruction on triangular meshes. The accuracy and well-balanced properties of the scheme are demonstrated in a variety of numerical simulations.

Several generalizations of this scheme are left for future research. This includes dry beds and dissipative systems.

Acknowledgments. We would like to thank Jonathan Goodman and Benoit Perthame for helpful discussions. 


\section{REFERENCES}

[1] P. Arminjon and M.-C. Viallon, Généralisation du schéma de Nessyahu-Tadmor pour une équation hyperbolique à deux dimensions d'espace, C. R. Acad. Sci. Paris Sér. I Math., 320 (1995), pp. 85-88.

[2] P. Arminjon, M.-C. Viallon, and A. Madrane, A finite volume extension of the LaxFriedrichs and Nessyahu-Tadmor schemes for conservation laws on unstructured grids, Int. J. Comput. Fluid Dyn., 9 (1997), pp. 1-22.

[3] E. Audusse and M.-O. Bristeau, Transport of pollutant in shallow water: A two time steps kinetic method, M2AN Math. Model. Numer. Anal., 37 (2003), pp. 389-416.

[4] E. Audusse, M.-O. Bristeau, and B. Perthame, Kinetic Schemes for Saint-Venant Equations with Source Terms on Unstructured Grids, INRIA report RR-3989, 2000.

[5] R. Botchorishvili, B. Perthame, and A. Vasseur, Equilibrium schemes for scalar conservation laws with stiff sources, Math. Comp., 72 (2003), pp. 131-157.

[6] R. Botchorishvili and O. Pironneau, Finite volume schemes with equilibrium-type discretization of source terms for scalar conservation laws, J. Comput. Phys., 187 (2003), pp. 391-427.

[7] A. I. Delis and Th. Katsaounis, Relaxation schemes for the shallow water equations, Internat. J. Numer. Methods Fluids, 41 (2003), pp. 695-719.

[8] K. O. FriedrichS AND P. D. LAX, Systems of conservation equations with a convex extension, Proc. Natl. Acad. Sci. USA, 68 (1971), pp. 1686-1688.

[9] T. Gallouët, J.-M. HÉrard, And N. Seguin, Some approximate Godunov schemes to compute shallow-water equations with topography, Comput. \& Fluids., 32 (2003), pp. 479-513.

[10] J. F. Gerbeau and B. Perthame, Derivation of viscous Saint-Venant system for laminar shallow water; numerical validation, Discrete Contin. Dyn. Syst. Ser. B, 1 (2001), pp. 89102.

[11] L. Gosse, A well-balanced scheme using non-conservative products designed for hyperbolic systems of conservation laws with source terms, Math. Models Methods Appl. Sci., 11 (2001), pp. 339-365.

[12] M. E. HuBbARD, On the accuracy of one-dimensional models of steady converging/diverging open channel flows, Internat. J. Numer. Methods Fluids, 35 (2001), pp. 785-808.

[13] G.-S. Jiang And E. TADMOR, Nonoscillatory central schemes for multidimensional hyperbolic conservation laws, SIAM J. Sci. Comput., 19 (1998), pp. 1892-1917.

[14] A. Kurganov and D. Levy, Central-upwind schemes for the Saint-Venant system, M2AN Math. Model. Numer. Anal., 36 (2002), pp. 397-425.

[15] A. Kurganov, S. Noelle, and G. Petrova, Semidiscrete central-upwind schemes for hyperbolic conservation laws and Hamilton-Jacobi equations, SIAM J. Sci. Comput., 23 (2001), pp. $707-740$.

[16] A. Kurganov and G. Petrova, Central-upwind schemes on triangular grids for hyperbolic systems of conservation laws, Numer. Methods Partial Differential Equations, 21 (2005), pp. 536-552.

[17] A. Kurganov and E. TAdmor, New high-resolution central schemes for nonlinear conservation laws and convection-diffusion equations, J. Comput. Phys., 160 (2000), pp. 214-282.

[18] R. J. LeVeque, Balancing source terms and flux gradients in high-resolution Godunov methods: The quasi-steady wave-propagation algorithm, J. Comput. Phys., 146 (1998), pp. 346365.

[19] R. J. LeVeque AND D. S. BALE, Wave propagation methods for conservation laws with source terms, in Hyperbolic Problems: Theory, Numerics, Applications, Vol. II (Zürich, 1998), Internat. Ser. Numer. Math. 130, Birkhäuser, Basel, 1999, pp. 609-618.

[20] S. F. Liotta, V. Romano, and G. Russo, Central schemes for systems of balance laws, in Hyperbolic Problems: Theory, Numerics, Applications, Vol. II (Zürich, 1998), Internat. Ser. Numer. Math. 130, Birkhäuser, Basel, 1999, pp. 651-660.

[21] H. Nessyahu AND E. TADMOR, Non-oscillatory central differencing for hyperbolic conservation laws, J. Comput. Phys., 87 (1990), pp. 408-463.

[22] B. Perthame and C. Simeoni, A kinetic scheme for the Saint-Venant system with a source term, Calcolo, 38 (2001), pp. 201-231.

[23] G. Russo, Central schemes for balance laws, in Hyperbolic Problems: Theory, Numerics, Applications, Vol. I, II (Magdeburg, 2000), Internat. Ser. Numer. Math. 140, 141, Birkhäuser, Basel, 2001, pp. 821-829.

[24] A. J. C. DE Saint-Venant, Théorie du mouvement non-permanent des eaux, avec application aux crues des rivière at à l'introduction des marées dans leur lit, C. R. Acad. Sci. Paris, 73 (1871), pp. 147-154. 